\title{
CoMoSy: a Flexible System-on-Chip Platform for Embedded Applications
}

\author{
Tran Van-Huan, Tran Xuan-Tu \\ SIS Laboratory, University of Engineering and Technology, VNU Hanoi \\ 144 Xuan Thuy road, Hano 10000 , Vietnam. Email thunity, tuxy@ @nu,eduvn
}

\begin{abstract}
Thanks to the rapid evolution of semiconductor technology, System-on-Chip (SoC) paradigm has become one of the most conmon design methodologits for quickly developing embedded systens to meet the high demands of embedded applications. In this paper, we present the desigu and implementation of a SoC platform targeted to controlling and monitoring applications. This proposed platform is composed of a 32-bit processor and a dozen of common lardware interfaces, providing the programmability and connectivity to peripheral devices such as memories, L.AN network, monitor, keyboard, ADC, DAC, or other LO devices, In addition, to increase the ffexibility of the system and to rapidly develop enduser applications, we also deploy an application-specific software framework with the robustness of a lightweight kernet and real-time applet mamagenent. The system model has finally been validated through the realization of a remote control camera system.
\end{abstract}

Keywords-System-an-Chip (SoC), embedded systems, FPGA-based design, microprocessor systems, embedded applications:

\section{INTRODUCTION}

N owadays, System-on-Chip (SoC) paradigm has 1 become very common in the design of embedded systems, allowing full sofwarehardware to be integrated into a single chip. In such embedded applications. SoCs should provide high functional Alexibility as well as processing capability [1]. Thanks to the cvolution of semiconductor technology, system designers can integrate more and more intellecture properties (IPS) into a system to meet the needs of applications, However, the growth of integration scale also leads to many new challenges in SoC design such as performance, on-chip communication, power consumption, and design cycles. These factors definitely depend on the design principles. design and implementation technologies.

To overcome these challenges, several ASIC platfoms of SoCs have been previously proposed in the literatures as well as commercial products. However, these platforms were developed for application-specific systems and they have limitations on flexibility and configurability. Even if we intend to improve those factors, it will make the sysiem more complex. In order to increase the flexibility and reduce the complexity of embedded systems, FPGA rechnology is obviously selected thanks to its advantages such as scalability, reconfigumbility, rapid prototyping, shon time-to-market, and low NRE (non-recutrent engineering) cost [2].

In fact, IPGAs have recently become very popular in implementing logic circuits. They can be used for many types of applications such as rapid prototyping platform [3], teleconmunications [4], digital signal pro. cessing [S]. The ffexibility and scaltubility of FPGAs have made them suitable for implementing embedded SoCs, where a complete system can be implemented on at single programmable chip. In this case, the soft-core processor provided by manufacturers can be reused for developing embedded systerns. This soft-core processor is a microprocessor which is fully deseribed in sotwuare; usually in an HDL (Hardware Description Language) and can be synthesized and implemented on FPGAs: One of the most advantages of the soft-cote processors 


\section{Research. Developmen and Application on Jiformation and Commanication Technology}

is that they can be easily customized to adapt the needs of a specific target application.

For those reasons, we have developed an FPGAbascd SoC plaifom, named as CoMoSy (Controlling and Monitoring System), using Xilinx MicroBlaze softcore processor. This platform is intently designed to be uscd for a large range of embedded systems in controlling and monitoring applications. With this platform, application mapping processes will be much easier and the production time will be significantly reduced. The proposed platform and its design methodology will be fully described in this paper, incliding both hardware and software issues.

The remaining part of this paper is otganized as follows Section II presents in detail the hardware architecture model and implementation of the proposed SoC platform Section III describes the application-specific software framework which is specially developed for the target hardware system. Section IV provides some main experiments and obtained results. Finally, futher discussions and conclusions ate given in section $\mathrm{V}$.

\section{PRoposED HARDWARE ARCHITECTURE - DESIGN AND IMPLEMENTATION}

\section{A. CoMosy architecture design}

As mentioned above, thanks to the flexibility and conligurability of FPGA technologies system designers have many choices in architecture design for their cmbedded systems. However, before mapping an application into a targeted FPGA technology, the first thing should be considered is the partitioning of software and hardware parts. The software part can be bult and executed on one or more processor cores if needed while the hardwate part is modularzed tnto particular functional blocks. These funcational blocks can be developed from the existing IP (Intellectual Properry) cores provided by FPGA suppliers or fully developed by designers in addition, an application platform may have more than one system configuration file (4.e. FPGA bitstream filc and software cxecution tile), allocated in different memon sections (using a ROM or a flash memory). They can be selected to configure/reconfigure the FPGA devich at power-on/nun-time for specific purposes: A bette lianware/software partitioning will produce a hight efficiency for the targeted embedded system.

The second thing is to select a proper FPGA device 4 order to fit the design. It means that the selected FPG H device does not only provide enough logic cells for the design but also has to meet the other requirements of th: design such as performance and processing capability For example; some Xilinx FPGA series can provid etther MicroBlaze softecore processor or PowerPC haro core processor $[6]$. In this case, a high-performano application might consider using PowerPC processe rather than MicroBlaze processor because the hard core processor is obviously better in performance tha the soft-core processor Otherwise, Microblaze soft-cor processor is more suitable for the applications in whic a higher level of Acxibility and configurability is needed

To develop enbedded systems for controlling an monitoring applications using Xilinx FPGA, we hav proposed a general architecture as described in Figure As requestod by targeted applications, this platform composed of three kinds of functional units processin units, data acquisition units, and data visualization unity The data acquisition units get data from either digit devices of analog devices (e.g a set of sensors) providing standard hardware input interfaces to haw connections with those devices, The MicroBlaze proces sor manipulates and processes the acguired data give out decisions to control the periperal devices: In add tion; some processed results are transferred to the dat visualization unils, where the dara can be represente in many ways (texi, graphic, light or sound) to bui Iriendly human-machine interfaces.

In general, CoMosy is a 32-bit bus-based platfor using a Microblaze soft-core processor $17 \mid$ as the centr processing unit. MicroBlaze is a RISC (Reduced Instny 


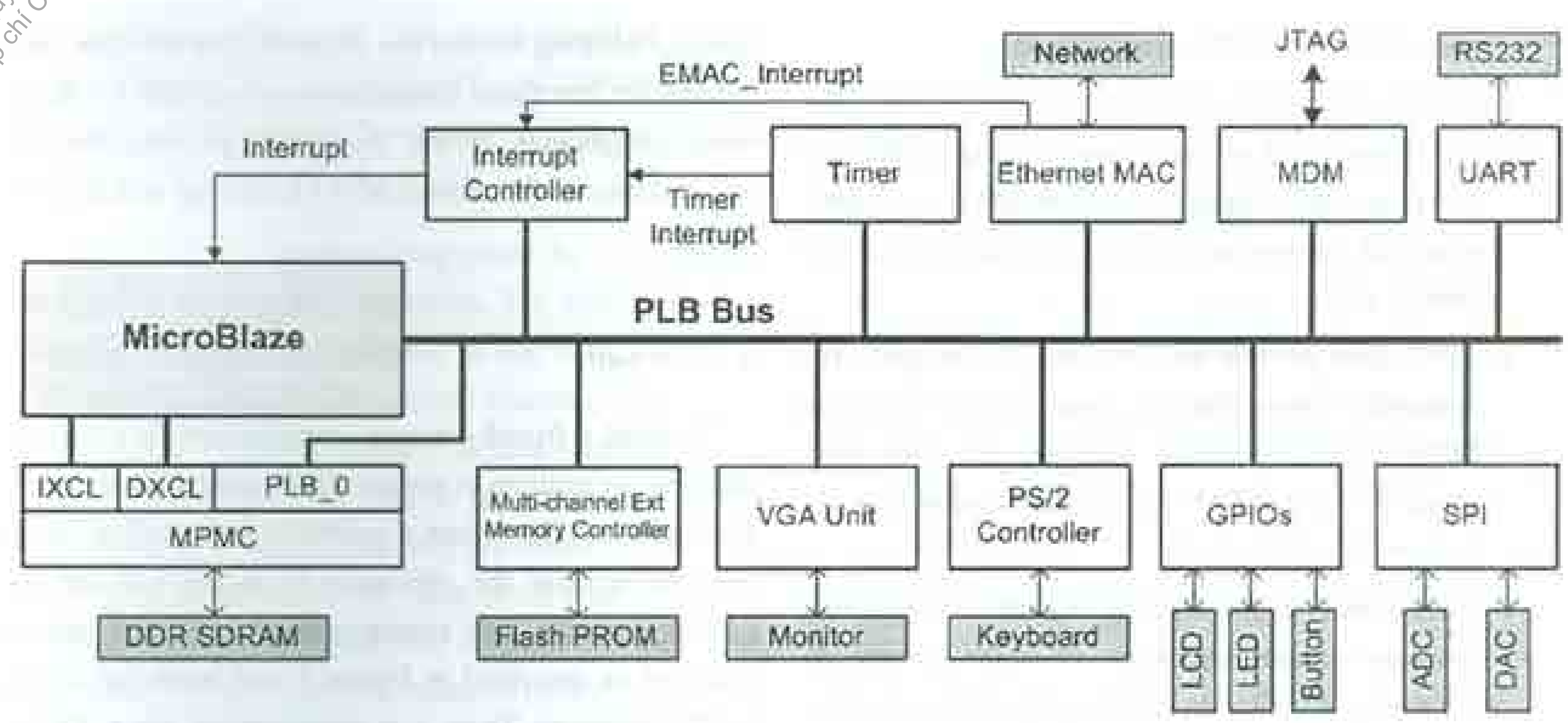

Fugure 1. General Architecture of CoMosy platiorm

tion Set Computer) processor that modelled in HDL. to be implemented on Xilinx FPGAs. Some optional funclion blocks integrated in this core are enabled to accelerate the processing ability such as Floating Point Unit (FPU), integer divider, integer multiplier, barrel shifter. "This processor core interacts with other IP cores through IBM CoreConnect PLB bus system [8], In addition to the processor, we have developed a dozen of IP cores. Some Xilinx IP cores have been reused to reduce design time and the others have been completely developed at the laboratory. The rest modules of the platform can be described as follows:

- MPMC core is a milti-port memory controller used to interface with DDR SDRAM. The MPMC unit provides three channels for independently accessing data on DDR SDRAM, where two of them serve as data cache channel and instruction cache chand of Microblaze processor, denoted as DXCL and $\mathrm{DXCL}$ respectively. The remaining channel (PL_B_0) provides a fast channel to transfer program data and instructions when executing. MPMC unit usually operates at a higher frequency than the processor core.

- Multi-channel externat memory controller tuit is another memory controller used to connect the system with a flash memory, This memory is intended to store the FPGA bitstream files and/or software execution files. It sometimes can be used as a file system device

- MDM (MictoBlaze Debug Module) unit is a hard. ware debug module used to debug the operations of MicroBlaze processor by using JTAG (Joint Test Action Group) interface.

- Timer unit is a 32-bit programmable interval timer which is required by almost software kemels for establishing software timers and task schedulers.

- Interrupt controller core handles the interrupt sig. nals arising from peripheral blocks and notifics the processor core by driving processoi's interrupt signal In the proposed platiom, there are two interrupt sources, timer internipt and Ethernet MAC interupt.

- Ethernet MAC unit is a 10/100Mbps Ethemet Media Access Controller. This controller is compatible 
with IEEE Std, 802.3 specification.

- UART, SPI, PS/2 units provide serial data links to connect with several devices such as RS-232 ports, analog-to-digital converters (ADCs), digitalto-analog converters (DACs), PS/2 keyboard, PS/2 mouse, etc.

- GPIO units provide bi-directional digital ports for discretely connecting to usual devices such as LEDs, LCDs, switches, buttons, etc. The portwidth of each GPIO unit can be customized by a parameter of the core.

- VGA unit is a special core which is completely designed at our laboratory in order to provide a friendly interface to VGA-compatible monitors. Although this unit is individually developed at the laboratory but it can be used for next FPGA-based system developments thanks to its compatibility to Xilinx FPGA design flow, The design of this unit will be described in detail in the next sub-section as a case study.

In the proposed platform, a master core (c.g. processor) accesses to slave cores using memory-mapped I/O methods. The address bus is shared for both memory controllers and the other slave devices. Thus, each unit in the system has its own address space determined by two parameters: base address and high address. The base addresses are automatically generated by Xilinx design tool while the high addresses are calculated from the memory/address size of IP cores and their corresponding base addresses.

As a simple architecture, the hardware model uses only one bus channel for interconnecting processing components (i.e. IPs) on the system. A new component can be easily integrated into the system thanks to the help of the design tools. Of course, the new component has to be compatible with PLB bus interface. However, the growth of the hardware architecture may affect the responise ability of the bus as well as the reliability of the system. In this case, system designers should consider creating several bus channels, for example, one channel for low speed components and another for highspeed components. Hence, the system is teally flexible and scalable on the change of the hardware architecture model:

\section{B. VGA tmit}

To build a friendly himan-machine interface for the platform, in addition to simple text-based LCDs we have developed a VGA monitor controller unit (called VGA unit) to increase the efficiency in displaying text as well as graphics. The architecture of this VGA unit is designed as described in Figure 2 and modelled using VHDL language. Then, it is implemented using Xilinx design flow [9] in order to make the design compatible to Xilinx design methodology, and therefore it can be used for the next designs. The VGA unit will display an image on the screen by driving the color signals and synchronization signals of the VGA interface according to the pixel data of the image.

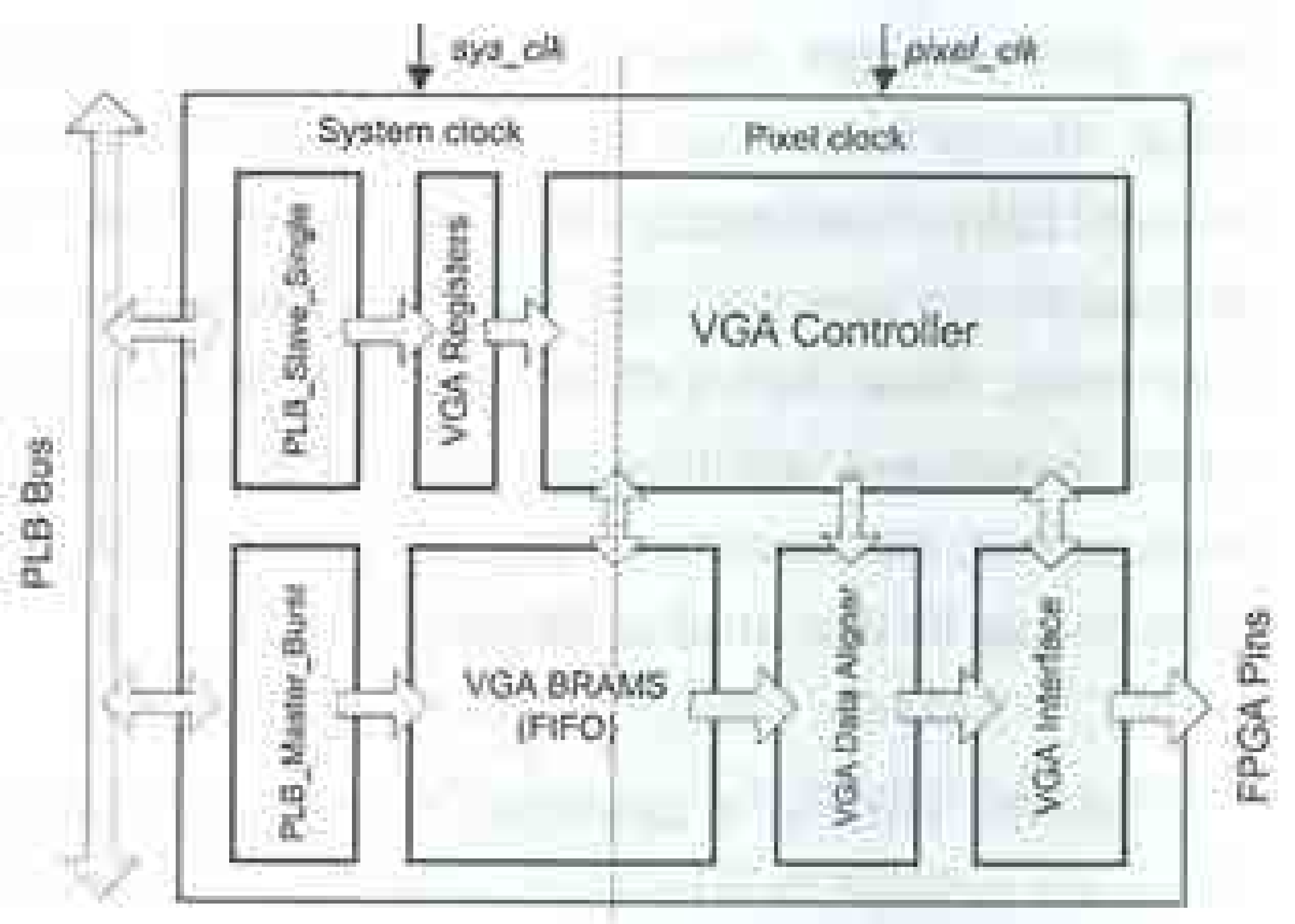

Figure 2, Proposed archilecture of VGA unit.

The size of an image is usually larger than memory resources available on FPGAs, for example, a $640 \times 480$ pixels at $24 b p p$ raw image has a size of 912.6 Kbytes while the Spartan-3E family has a limit of 81 Kbytes block RAM [10], the image therefore cannot be entirely 
20 stored inside the VGA unit. The image should be held on an off-chip memory (e.g. SDRAM) and transferred into this VGA unit by small data blocks during the display time.

There are two solutions that could be addressed to handle the data transfer operations. The first solution is to attach a bus master interface to the VGA unit to be able to access data on the extemal memory. The second one is to use a Direct Memory Access (DMA) core to co-operate with the VGA unit. In the second case, VGA unit does not need to include any bus master interface, it receives the data transmitted from DMA core. However, the processor has to initiate the operation of the DMA core whenever the VGA unit wants to refresh the screen. For that reason, we prefer the first solution to gain the flexibility and independency of the VGA unit.

One of the most challenges in designing VGA unit is to detcrmine the depth of memory buffer within the core and the buffering strategy because these parameters will affect the cost-efficiency and display quality of the system on a specific display resolution. In this work, we have taken into account these constraints for VGA standard. In consequence, the architecture of VGA unit (presented in Figure 2) is composed of:

- PLB Master interface with burst transfer mode is usually used for transferring data from the external memory to FIFO block:

- PLB Slave interface serves the reading/writing operations between the processor and VGA unit.

- Three 32-bit registers contain the command data and the properties of the images such as width; height, base address.

- VGA BRAMS/FIFO is a 256 bytes dual-port memory buffer for storing a bulk of data:

- VGA Data Aligner synchronizes data between FFFO block and VGA Interface block.

- VGA Interface drives the synchronization signals of the monitor.
- VGA Controller is reponsible for VGA timing and the operations of the whole VGA unit

There are two clock domains in this design: system clock (sys_clk) and pixel clock (pix_clk). The pixel clock absolutely depends on the display resolution and the refresh rate of the screen specified by standards (see Table 1). Even though two clock signals are independent, the system clock must be higher than the pixel clock to ensure that the appearance is smooth (without flickering) and the overhead on bus traffic is eliminated.

Table 1

DISPLAY STANDARD SPECIFICATION

\begin{tabular}{|l|c|}
\hline Display standard & Pixel frequency $(M H z)$ \\
\hline \hline VGA $640 \times 480960 H z$ & 25.175 \\
\hline SVGA $800 \times 600960 H z$ & 40.000 \\
\hline XGA $1024 \times 768960 H z$ & 65.000 \\
\hline WXGA $1280 \times 800960 H z$ & $\$ 3.460$ \\
\hline
\end{tabular}

The VGA unit is then modelled in VHDL language and simulated by using ModelSim simulator (Mentor Graphics). The synthesis results on Spartan-3E device show that the VGA unit can operate at a speed up to $160 \mathrm{MHz}$. The unit is thus possible to provide higher resolutions thin VGA standard such as XGA or WXGA $1280 \times 800$. The detail design and implementation of the VGA unit has been reported in [11].

\section{Implementation}

In this section, we present the implementation of the proposed platform on Xilinx Spartan-3E device to evaluate some typical metrics of the system. From the system specifications described above, the design and implementation can be completely managed by Xilinx Platform Studio environment [9]. All required peripheral hardware blocks are integrated into the system and they are configured to a proper operation mode. Then, we let the tool automatically perform synthesizing and 
implementing processes with provided input consiraints. Finally, the design is verified again in order to check whether it meets the system specifications, fiming and power consumption issues before being loaded into FPGA devices.

Table II shows the resource utilization on Spartan3E XC3S500E device; distributed on the logic cells (including slides and look-up-tables); Input/Output pads (I/Os): primary RAM blocks (BRAMs): Digital Clock Manager blocks (DCMs); and hard-macro multipliers (MULTs). Obviously, the whole design is fit on Spartan$3 \mathrm{E} \times \mathrm{XC} 3500 \mathrm{E}$, most logic elements and BRAMs are used (more than $80 \%$ of available resources). The design also meets the timing constraints to operate at $50 \mathrm{MHz}$.

Table II

RESOURCE UTH ZRNION (SPARTAN-3E-XC3S500E)

\begin{tabular}{|l|c|c|c|}
\hline Name & Available & Used & Utilization \\
\hline \hline Logic & 9312 & 7616 & $82 \%$ \\
\hline WOs & 232 & 86 & 375 \\
\hline BRAMs & 20 & 19 & $95 \%$ \\
\hline DCMs & 4 & 2 & $50 \%$ \\
\hline MUITs & 20 & 7 & $35 \%$ \\
\hline
\end{tabular}

In addition to hardware overhead, power consumption is another important parameter in embedded system design. The power consumption of our system is estimated using XPower Analyzer tool. Table III summarizes iwo metrics of power consumption, total quescent power and tolal dynamic power. Because we implement our design ising FPGA technologies, the only concerned parameter is the dynamic power of the design. Most of the quiesoent power depends on the target technologies and we cannot change.

The distribution of power consumption of the design is also shown in Figure 3 . The power consumed on T/Os is the most considerable $(80 \%$ of the whole system
Tabie III

PownR Consunhtios

\begin{tabular}{|l|c|}
\hline Type & Power (mV) \\
\hline Total quescent power & 105 \\
\hline Total dynumie power & 643 \\
\hline Totil consumed power & 748 \\
\hline
\end{tabular}

power consumption) because the DOS usually require ligher current than the other entities.

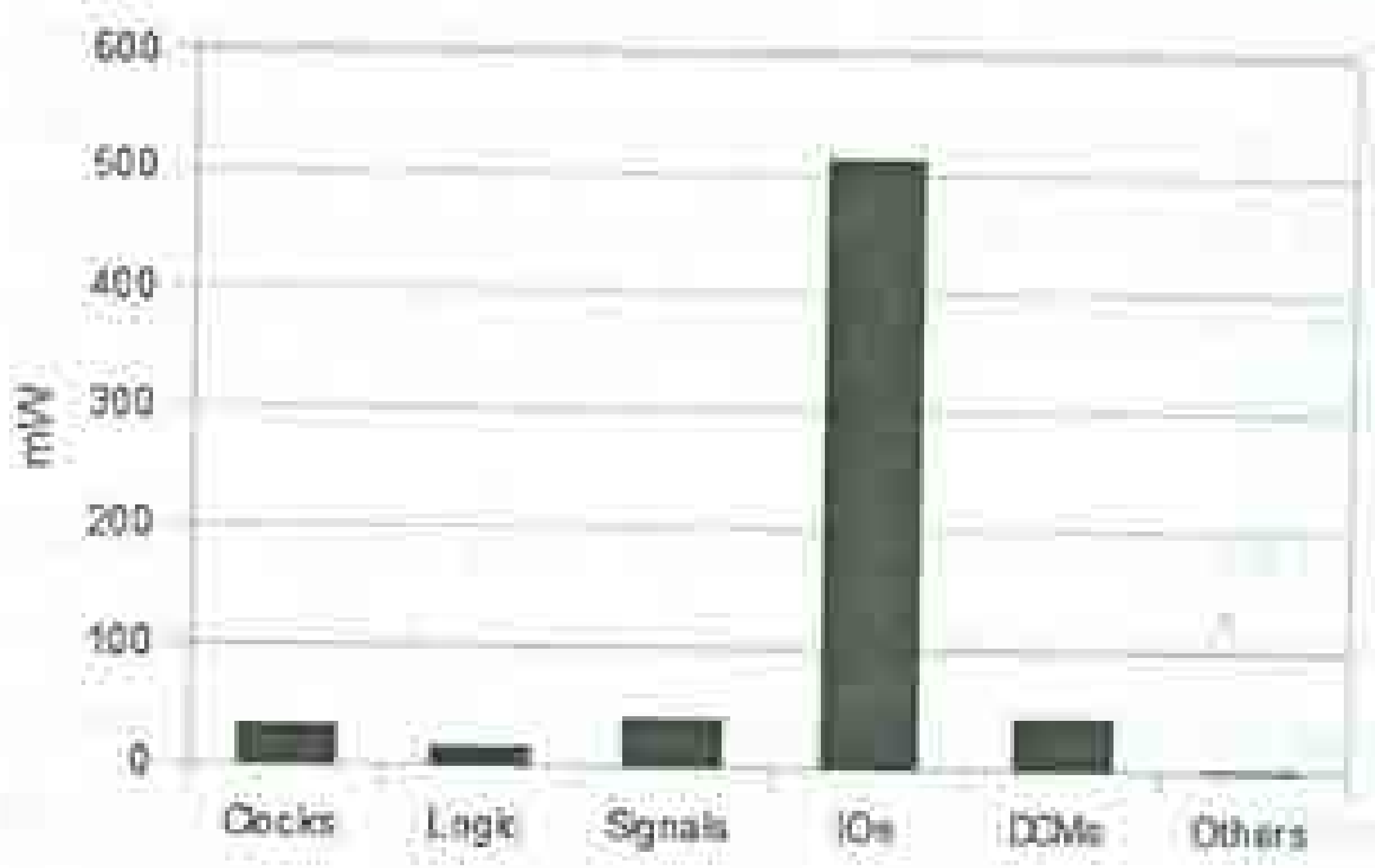

Figure 3. Power consumption distritution.

\section{SOFTWARE SYSTEM}

In order to adapt to the change of the hardware architecture, we develop a scalable and rehable software framework for the proposed hardware. The main purpose of this framework is to provide mechanisms for managing hardware resources and uset applets. To do this, we has adopted several open software libraties. modiffed and integrated them to build the software framework as presented in Figure 4. In this software framework, the most important part is the kemel based on Xilkemel [12]. This kemel can support the most common POSIX APIs (Portable Operating System Interface - Application Programming Interface) [13] such as thread management, thread synchronization services, and thread communication services. 


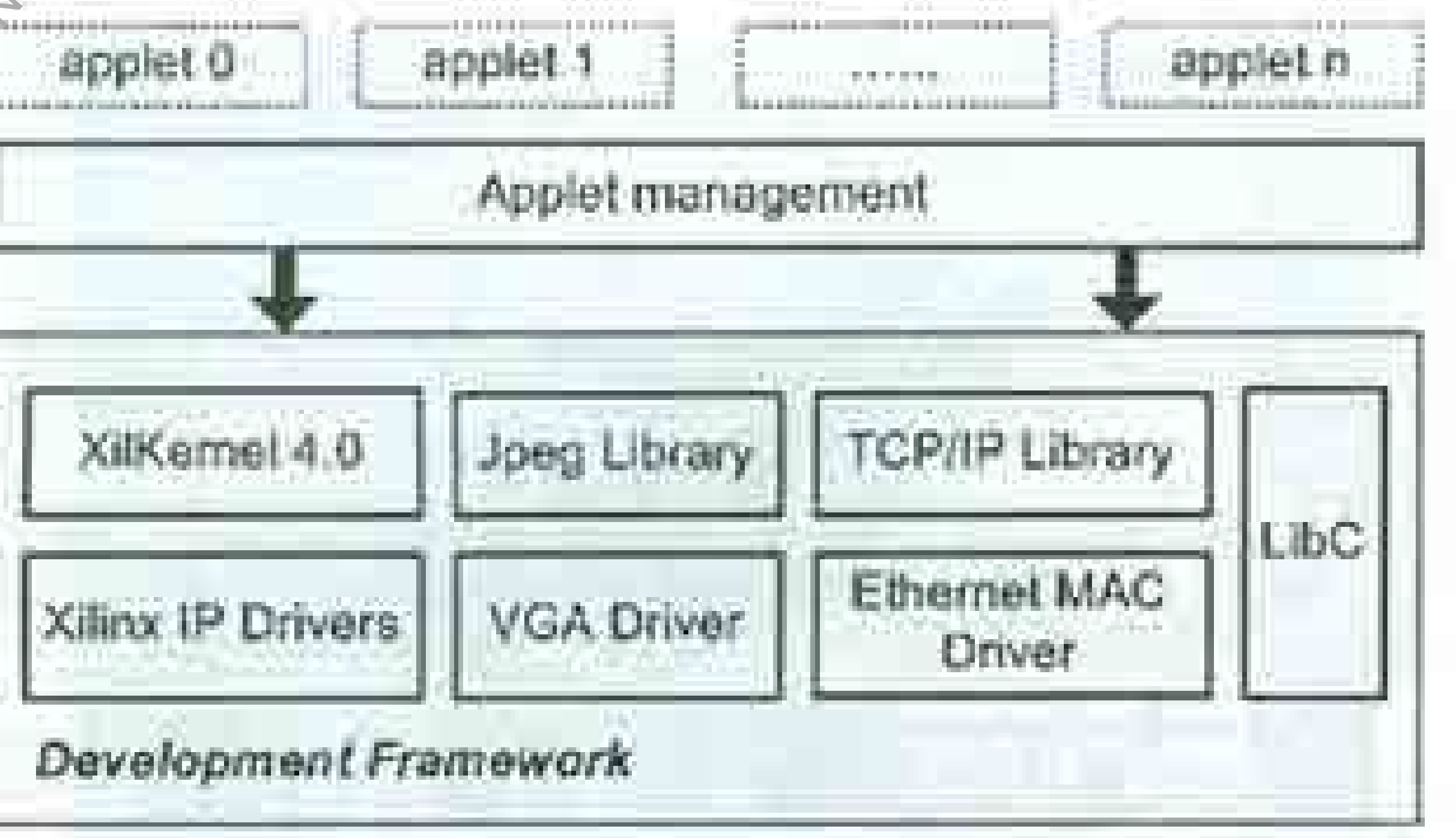

Figure 4. Softward development framework.

In this software framework, the lowest layer is driver layer. This layer is composed of Xinx IP drivers (used for Xilinx IP cores) and VGA driver (used for our hardware design of VGA init). Upon the drivers is the kemel and library layer. In this layer, beside the kerriel We integrate several popular libranes such as standard C. library, JPEG decoder library [14], TCP/P protocol stack library [15] to help software designers to quickly build their applications, In higher layers, we develop an applets management layer to handle all user applets: Each applet is a simall application performing a specific function, described by the following object:

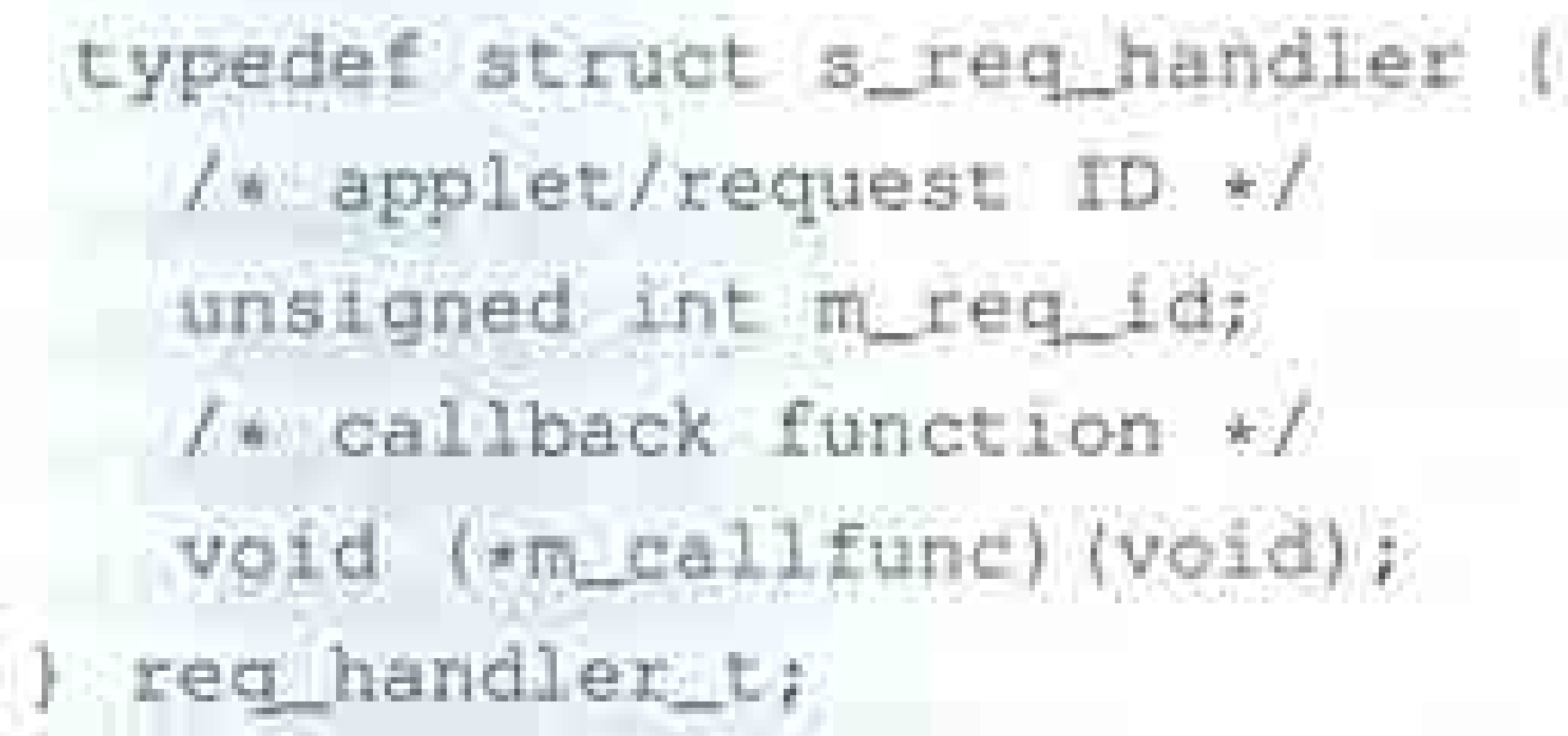

In addition, to help the users to easily interact with the system, the applets management layer is designed to be able to create a command-line interlace where users can type a command to execute the corresponding applets. The applets managentent layer has been built with three threads: GPIO handler thread, keyboard handier thread, and request handler thread (see Figure 5). The GPIO handler thread performs monitoring states of digital imput ports and maps each state to a specific re- quest ID (identification). Similarly, the keyboard handler thread receives user commands from keyboard and maps each of them to a specific request ID. These request IDs are then sent to the request handler thread to specify which applet would be invoked. Thanks to this software model, software designers are able to quickly develop end-user software applications and embed them to the rest of system without considering the reliability of the sofiware system.

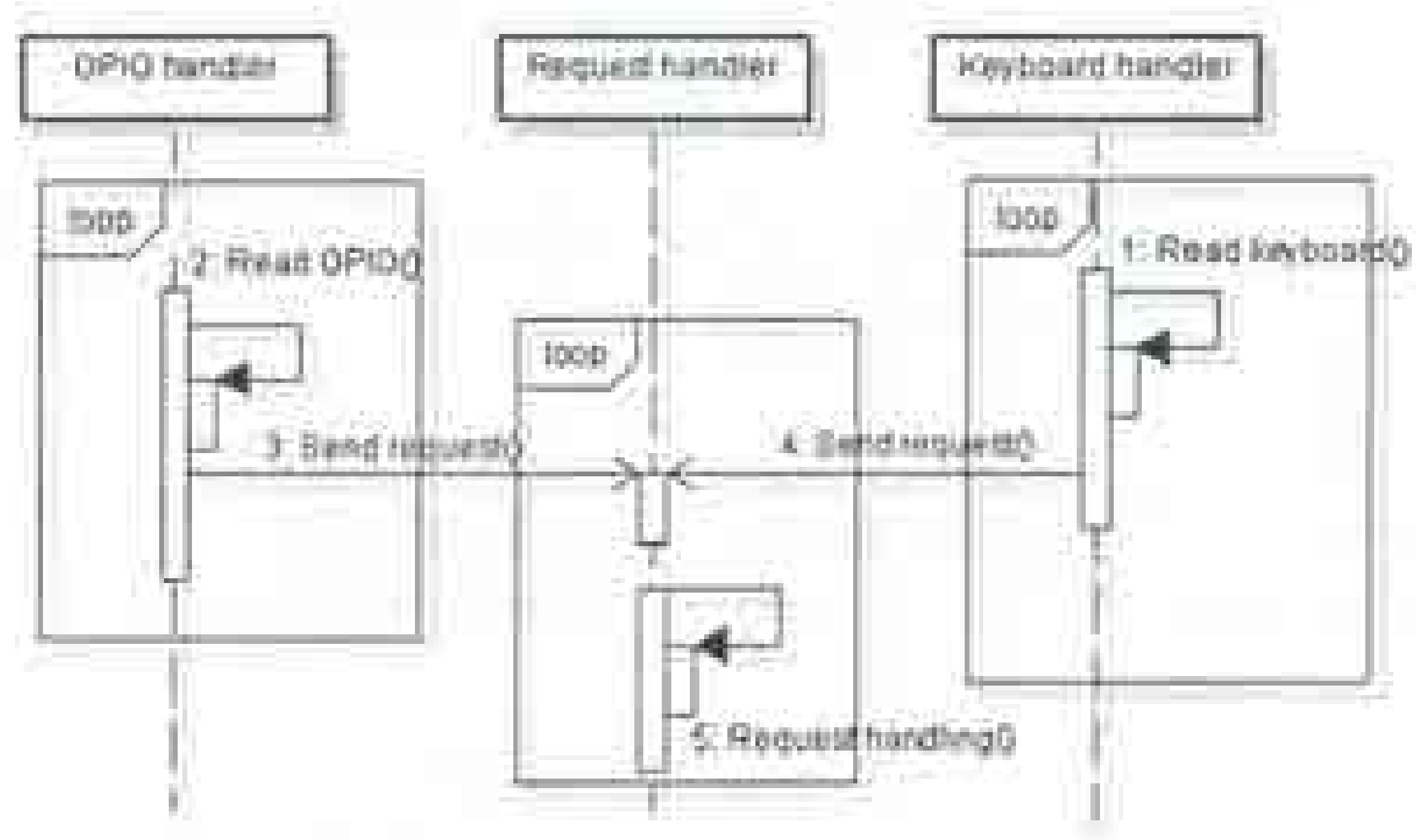

Figure 5. Sequence diagnam of the applet managemen.

\section{EXPERIMENTS}

Verification and validation are two key processes in system design flow and take much time in the design cycle. The using existing resources (including IP cores and software libraries) provided by EDA tools and open source community not only helps system designers save time but also improves the stability and reliability of targeted systems. At a higher level, we present an experiment to verify hardware/software models and their corporations in the platform by developing a remote camera application. In this context, we implement the entire CoMoSy platform on Spartan-3E Starter Kit [16]. This board is based on Spartan-3E FPGA devices with all necessary peripherals for the application such as $512 M$ bits SDRAM, 128 M bits PROM, Ethernet PHY controller, LCD, LEDs, buttons, etc. PS/2 port and VGA port are also connected to a PS/2 keyboard and monitor as standard inputoutput devices; 


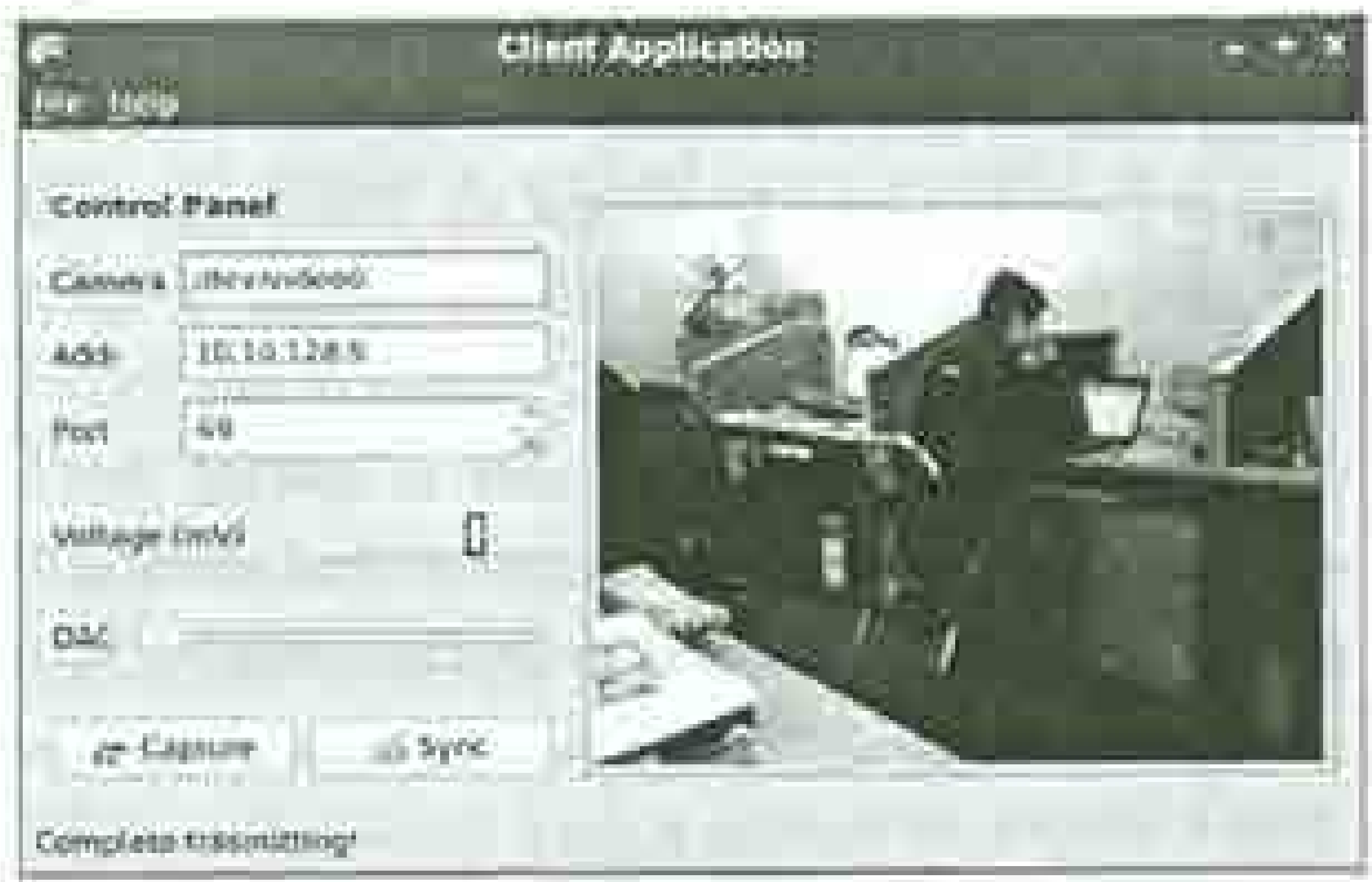

(a)

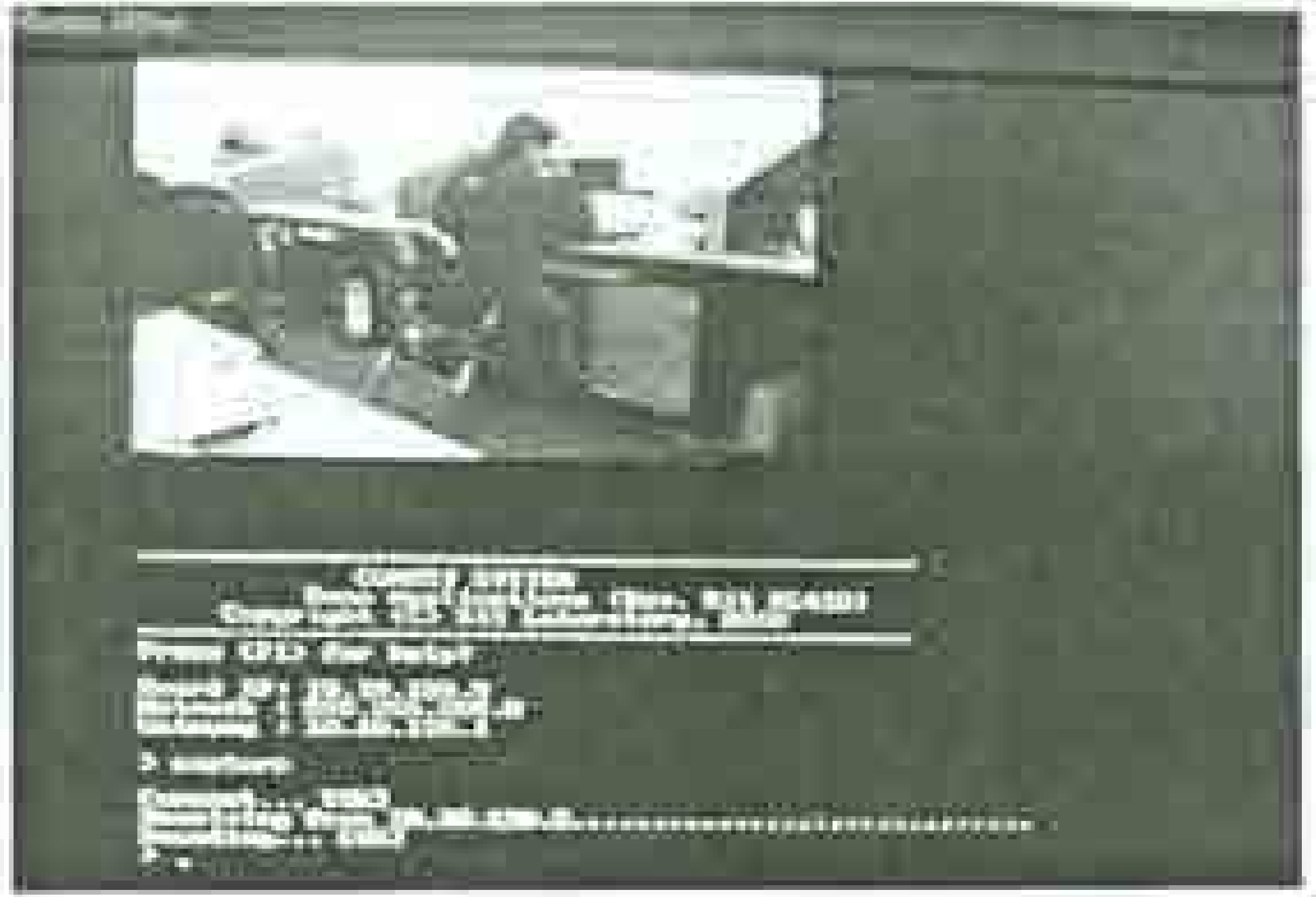

(b)

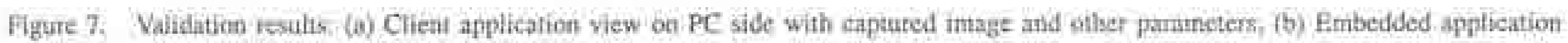
view on CoMosy tide will decoded inage.

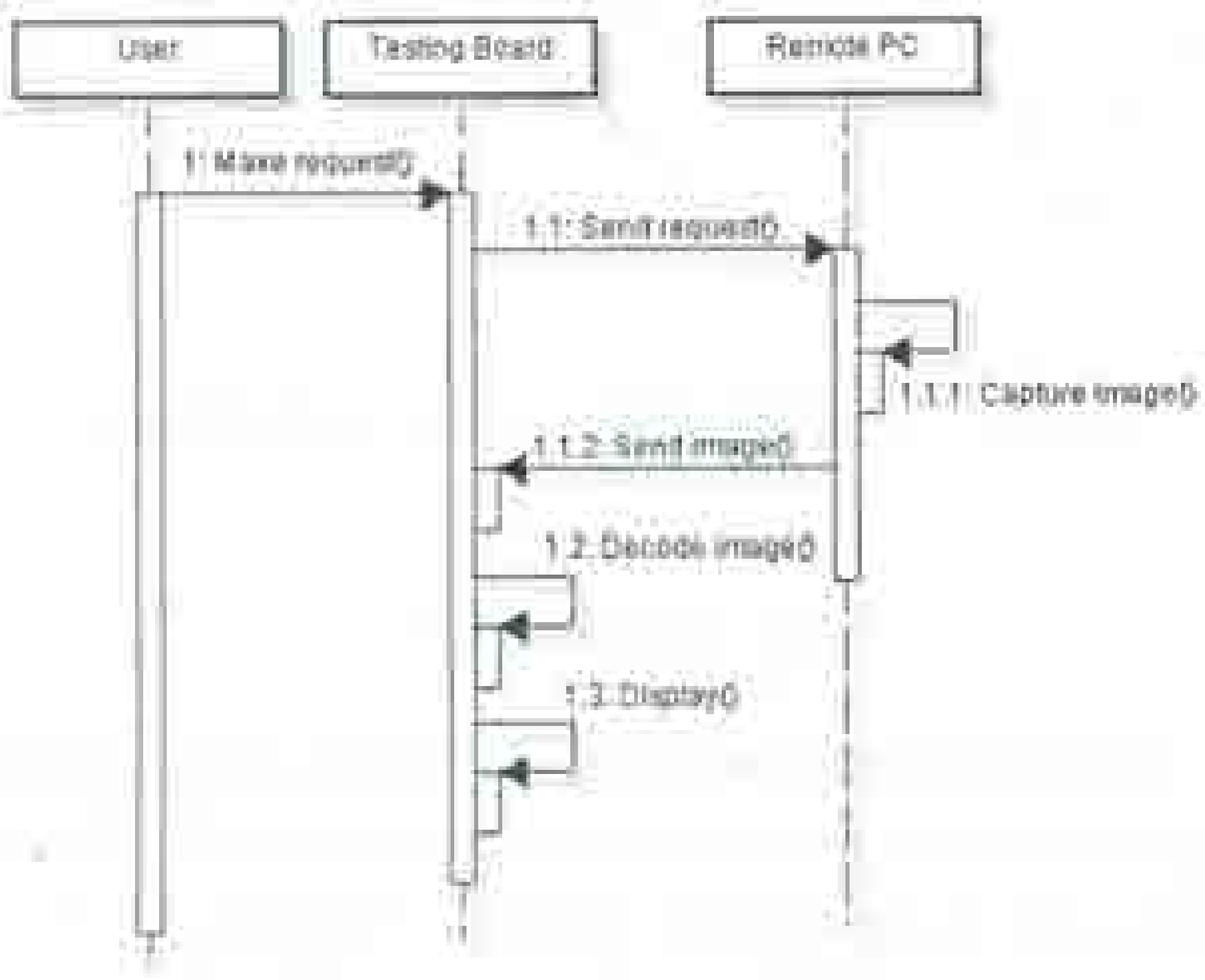

Finute 6. Scupeice diagram for the remole camera application.

A desktop PC running Linus operating system is used to establish communication with the FPGA board. A camera is comected to this PC wia USB interfaces. Transferting data between $P C$ and the testing board is done on $\mathrm{LAN}$ network thanks lo the integraled Ethernet MAC coniroller as mentionined above. Figure 6 presents the secuence diagran for the renote camera application.

The scenario is that the users first make a capturing request on the testing board, and then the resting board sends this request to the remote $\mathrm{PC}$ through $\mathrm{LAN}$ network. Whenever PC recelves a proper request, it captures an image from camera encodes the image and then sends it back to the testing board. After completely recerving the image, the resting board will decode and display the decoded image on the screen. The system provides several ways lor the users to invoke the capturing request, either lyping a console command of pressing it key binding or hitung a button on the board. So that. it makes the system more Hexible in interacting with the users.

Such system requires two application softwares to handle all actions on PC and on the testing botrd. The embedded application rumning on the testing board is butl with the APls provided by the software system. The $\mathrm{PC}$ application software is whtten in $\mathrm{C}++$ language using the buill-in classes of QI Designer $[17]$ and Videoz inux. library [18]. Inter-process communicauon between two applications is based on socket mechanism. Figure 7 presents the results of our experment the captured. image is shown on both PC application and the teating bourd (in 24-bit cotor mode) with Xilinx Virex-4 FPGA development kit.

On the stme testing model, we also make other experiments to validate the operation of analog-to-digital 


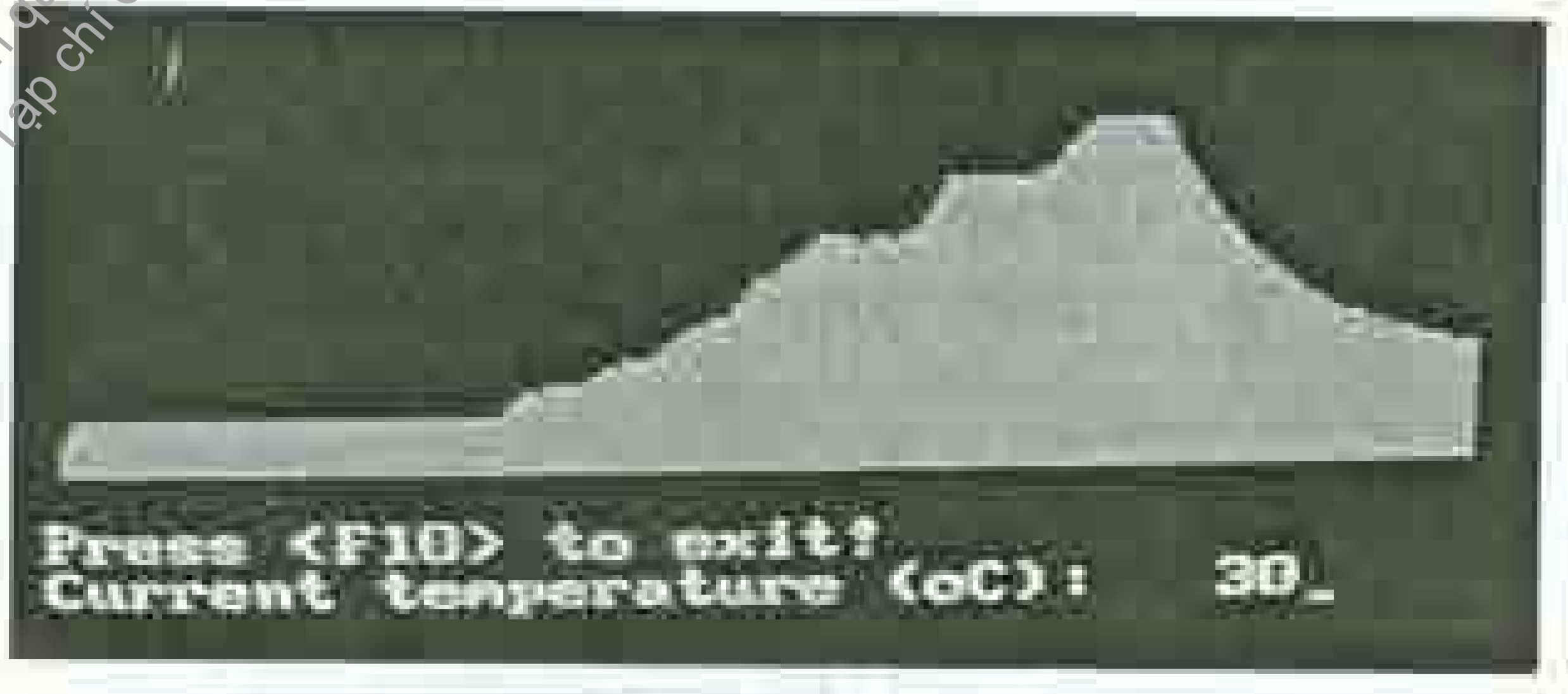

Figure 6 : Tempersune mpoitoring application

converter (ADC), digital-to-analog converter (DAC) via a shared SPI interface. One of these applications is the temperature monitoring application. In this application, a temperatire sensor is connected to an ADC. This ADC is Interfaced with the system by asing SPI anit. The temperature variation is displayed on the testing board as presented in Figure 8 .

Due to the logical resources of Spartan-3E device, the perfomance of CoMoSy achieved on this board is limited, it may take a few seconds to completely decode 1. JPEG inage with the frame size of 320 × 240 pixels. By implementing on high-performance devices or higher integration density devices (Virtex-1I or Virtex-4 for example), the system performance can be significantly improved through optinization processes and the recon= figurability of MicroBlaze processor Nevertheless, these experiments have approved the functionalities as well as the applicability of CoMoSy platform for controlling and monitoring applications.

\section{Conclusion}

In this paper, we presented the design and implementation of a flexible System-on-Chip piaform for controlling and monitoring applications using Xilinx. FPGA technology, both software and hardware issues. The proposed platform uses a 32-bit MicroBlaze softcore processor and PLB bus as the base system, We have integrated several hardware uinits/nterfaces to provide functional capabilities for a wide range of embedded applications, including GPIOs. SPIs, UART, PS/2. DDR
SDRAM, Ethemet MAC, and VGA unit In particilar, the VGA unit is an in-house design but it is compatible with Xlinx design flow and therefore can be used for other designs. This VGA unit supports malti-resolution displays. The sofiware system is intently developed from existing resources, including a lightweight kernel. IP core drivers, TCP/P protocol stack library JPEG decoder library to let users build their softwate applications guickly at high abstraction level.

\section{ACKNOWLEDGMENI}

This work is partly supported by Vietnam National University, Hanol (VNU) under the research project No. PUF.08.06.

\section{REPERENCES}

[1] Yen-Kuang Chen and Sun Yuan Kang. Ttend and Challenge on System-on-a-Chip Designs Jaurat of Signal Processhy Sysems. $53(1-2)=217-229,2008$.

(2) Gres Martin Plaform ASICs yersu FPGA In LSI logic Comoratiot, September 2005.

(3) Donink Langen, Jorgechristian Niemam, Mario Porrmanti, Heikn Kalte, and Ulinch Ruckert. Implementation of a RISC Processor Core for SoC Designs - FPGA Prototype us ASIC Implementation In Procedding of the IEEE:Wontolop: Hefmosenous rotonfigurable Systems on Chip (SoC). Hamburg. Germainy. 2002 .

[4] P. Murphy, F, Lou, A. Sabharwal, and J Patrick Fraitz. An FPGA Based Rapid Prototyping Platform for MIMO Systems. In Proceedings of the $37 \mathrm{~h}$ Antomar Conference on Signats Systems and Computers, pages 900-904. Pacific Grove, CA. November 2005.

[5] Chr-jui Chou, Sarth Mohanuknstman, and Joseph B. Evars. FPGA Implemenulion of Dighal Fiters. In Procedings of the 4h themanonat Conferance on Signal Processing Applications and Techology, pages 80-88, 1993.

(6) Xilim Corpuation Virter-4 Fanily Overvewi Data Sticet User Ginde, 2007.

17 Xilins Comporation MicroBlaze Processor Reference Goido User Gude, August 2008.

[8] Xiiin Corporation PL BV 46 Interface Simptifications (v1.0) User Guide, Ociober 2007 .

[9] Xilinx Conporation Embedled Systam Took Reference Guide User Guld, June 2009

[10] Xilin Corporation Spatta-3E PFGA Family. Data Shect User Guldr. August 2009. 
[11) Van-Huan Tran and Xuan-Tu Tran An Efficient Architecture Design for VGA Monitor Controller. In Proceedings of the Intemational Conference on Consume Electronics, Communications and networks, pages 3917-3921, Hubel, China, April 2011.

[12] Xilins Corporation OS and libertes document and collection. User Guide, June 2009.

(1) IFFE. POSIX: Pottable Operating System Interface. Standard Specification, 2006.

(14) Lue Saillard. Tiny Jpeg decoder, Technieal report, 2007.

[15] Adam Dumkels. Design and implementation of the lwiP TCP/P stack Technical report, February 2001.

46i Xilins Corponation-Spartan-3E Stan Kit Board User Guide User Guide, 2009.

117) Nokia Q1 - Cross-platform application and UI framework Technical report, 2010

[18] Gerd Knar VideotLinux. Technical report.

[iv] SECONS Led VGA Signal Timing, Technical report, 2008.

\section{AUTHORS' BIOGRAPHIES}

Tran Van-Huan was born in Vietriam, in 1985. He received the Bachelor of Science in Electronics. and Telecomminication from University of Engineering and Technology, Vietnam National University, Fanoi (VNU UET) in 2007 . He is currently working as a researcher of the Key Laboratory. for Smart Integrated Systems - VLSI Systems Design group, University of Engineering and Technology, VNU Hanot. He has itrvolved in CoMoSy, VENGME projects for embedded systems and multimedia applications. His research interests include System-on-Chip design and verifica- non, FPGA-based desipi, embedded systens, VLSI systensfercuits design for mulimedia application. Xuan-Tu Tran wus born in Nghe Ant, Viet-tian, in 1977. He recelved a B.Sc degree in 1999 from Hanol University of Science and a M.Sc degree. in 2003 from Vienam National University, Hanoi; all in Electronies Engneet - ing and Communications; and a Ph.D. degree in 2008 from the CEA-1.ET, MNATEC (in collaboration with Grenoble NNP), France in Micro Nano Electronics.

Tran Xuin-Tu has worked as a lecturet at Vietoani National University, Hanoi (1999.2003), as a research engineet at the CEA- LETI, MINATEC; France (20032008), and then as an assistant professor at the University of Engineering and Technology (UET), VNU Hanol (2008-recent). He was a visiting/invited professor al the Lnversity Paris-Sud 11. France $(2009,2010)$, visiting professot at Grenoble INP in 2011. He is currently. Deputy Director of the Key. Laboratory for Smart Integated Systems and head of VLSI Systens Design group. He is in charge for CoMoSy. VENGME projects for cmbedded systems and multimedia applications: His rescarch interests inetude design and test of system-on-chips, nework-on-chups design-fortestability, asynchronous/synchronous VLSI design, and hardware architectures for multimedia applicaltons. He is a member of the 1EEE, IEEE CAS, and the Executive Board of the Radio Electronics Association of Vietram (REV). 